\title{
Vibratory and perceptual measurement of resonant voice
}

\author{
Yiu, E. M.-L $\mathrm{L}^{1,2}$ \\ Chen, F. C. ${ }^{1}$ \\ Lo, G. ${ }^{1}$ \\ $\&$ \\ Pang, G. ${ }^{1}$
}

Voice Research Laboratory ${ }^{1}$

Division of Speech \& Hearing Sciences

The University of Hong Kong

Pokfulam HONG KONG
Visiting Professor ${ }^{2}$

Discipline of Speech Pathology

Faculty of Health Sciences

The University of Sydney

NSW AUSTRALIA

Corresponding Author: Professor Edwin Yiu, Voice Research Laboratory, Division of Speech \&Hearing Sciences, The University of Hong Kong, 5/F Prince Philip Dental Hospital. Email<eyiu@hku.hk> 


\begin{abstract}
Purpose: This study set out to investigate whether there existed a correlation between auditory-perceptual judgment of resonant voice and the physical vibration in the facial bone. The magnitude of bone vibration in the facial bone during different types of voice production (resonant versus non-resonant versus strained voices) was also investigated.
\end{abstract}

Method: Thirty-six healthy normal subjects produced three types of phonations: resonant voice, habitual non-resonant voice and strained voice. A piezoelectric accelerometer was used to measure the vibrations in the nasal bridge and the peri-laryngeal area during the phonation. Seventy-two selected nasal sounds (/ma/) produced under the resonant voice condition by these speakers were rated by two experienced speech pathologists on the magnitude of auditory-perceptual resonance using an 11-point equal appearing interval scale. The magnitude of bone vibration was also compared across the three voice types.

Results: Significant moderate correlations were found between the physical bone vibration and the auditory-perceptual rating of resonant voice at the nasal bridge of the facial bone (0.6). Resonant voice, when compared to the strained and non-resonant voice types, was also found to have a significant increase in the magnitude of facial bone vibration. Nasal stimuli, when compared to non-nasal stimuli, facilitated greater bone vibration during resonant voice production.

Conclusion: Piezoelectric accelerometer, as an objective quantitative tool, measures reliably the extent of bone vibration in resonant voice phonation. It is a useful tool for determining the bone vibration in relation to resonance voice.

Keywords: humming, vibration, accelerometer, resonant voice, resonance, perceptual voice 


\section{Vibratory and perceptual measurement of resonant voice}

Resonant voice training is widely used in facilitating voice projection using a relaxed phonatory posture with minimal effort (Jacobson \& White, 1994). The theory behind this particular voice production method is that the vibratory pattern of the vocal folds creates minimal impact force between the two vocal folds (Verdolini, 2000; Verdolini, Druker, Palmer, \& Samawi, 1998; Verdolini, Hess, Titze, Bierhals, \& Gross, 1999). Resonant voice is also commonly used as an intervention technique to treat individuals with voice problems (Boone, McFarlane, \& Von Berg, 2005; Colton \& Casper, 1996; Cooper, 1977; Verdolini, 1998; Yiu, 2008). Nasal stimuli $(/ \mathrm{m} /, / \mathrm{n} /$ or $/ \mathrm{y} /)$ are often used as the initial practice items to facilitate resonant voice training (Boone, et al., 2005; Verdolini, 2000; Yiu, 2008). The training procedure generally requires the learners to make use of their auditory-perceptual and tactile feedbacks to monitor their resonant production (Behrman and Haskell, 2008). The use of auditory-perceptual feedback per se may be difficult for a novice learner as it has been shown that even trained listeners could only identify accurately $74 \%$ of resonant voice samples among non-resonant voices using the auditory-perceptual method (Barrichelo \& Behlau, 2007). Such difficulty could be attributed to the fact that auditory-perceptual evaluation of resonant voice is a subjective evaluation process that involves a reliability issue. Accurate auditory-perceptual identification of resonant voice depends on a number of variables. These include the listeners' memory of a specific voice quality signal, acoustic context of the voice stimuli and prior knowledge and experience in dealing with voice quality (Kreiman, Gerratt, Kempster, Erman, \& Berke, 1993; Shrivastav, Sapienza, \& Nandur, 2005). Therefore, auditory-perceptual learning of resonant voice is not necessarily an easy procedure, especially for inexperienced learners. Indeed, among the different means of feedback, resonant voice training has traditionally put more emphasis on using tactile sensation (Behrman \& Haskell, 2008) at the anterior skull bone (maxillary) or alveolar ridge inside the oral cavity (Verdolini, 2000; Verdolini-Marston, Burke, Lessac, Glaze, \& Caldwell, 1995). Tactile sensation of the vibration is regarded as a good supplementary strategy in learning to acquire resonant voice. 
It has been suggested that the physiology of resonant voice production involves barely adducted or slightly abducted vocal folds during phonation (Verdolini, et al., 1998; Verdolini, et al., 1999). When used as a therapy technique, this laryngeal posture is believed to reduce the overall vocal effort, thus allowing lesions, if any, on the vocal folds to take time to heal (Chen, Huang, \& Chang, 2003; Roy, et al., 2003; Verdolini-Marston, et al., 1995).

A number of studies have investigated the outcome of resonant voice training and evaluated the general voice condition following resonant voice therapy using auditory-perceptual voice quality evaluation (Chen, Hsiao, Hsiao, Chung, \& Chiang, 2007; Verdolini-Marston, et al., 1995; Yiu \& Ho, 2002), acoustic quality changes (Chen, et al., 2007; Yiu \& Ho, 2002), aerodynamic analysis (Chen, et al., 2007), vocal fold vibratory pattern (Barrichelo \& Behlau, 2007; Chen, et al., 2007), and speakers' self perception of functional impact using the Voice Handicap Index (VHI) (Chen, et al., 2007; Verdolini, 2000). It should be noted that these studies only measured the general voice quality before and after the resonant voice training and not specifically measuring the resonant voice itself. Indeed, there are very little, if any, quantitative data on the resonant voice itself in the literature, with the exception of an aerodynamic study by Peterson, Verdolini-Marston, Barkmeier, and Hoffman (1994), and an acoustic study by Titze (2001) using a physics model.

This study set out to explore whether bone vibration measured by a piezoelectric accelerometer during resonant voice production could reflect the magnitude of resonant voice. The piezoelectric accelerometer is a vibration sensor using piezoelectric effect that converts mechanical energy into electrical energy. Using such an objective measurement technique to quantify the tactile sensation would definitely have a greater advantage in addition to the use of the auditory-perceptual judgment method that has a relatively low reliability.

Accelerometer has been shown to be useful in measuring certain aspects of voice production as it is not easily affected by environmental noise (Svec, Titze \& Popolo, 2005). Accelerometer has been used in the peri-laryngeal (Svec, Titze \& Popolo, 2005; Stevens, Kalikow and Willemain, 1975; Sundberg, 1983), nasal (Stevens et al, 1975; 
Horii, 1983, Lippmann, 1981), and sternal areas (Sundberg, 1983) to measure the vibration in the bone and skin layer.

Sundberg (1983) investigated the accelerometric vibration in the sternum, thyroid cartilage and below the larynx in singing voices. He found the extent of vibration detected by the accelerometers at these sites is a good reflection of the amplitude of the fundamental frequency of the voice source. Stevens et al (1975) found the vibration measured by the accelerometer at the nasal area was affected more by the amount of nasality than by the overall amount of voice intensity. Indeed, Lippmann (1981) found that the lateral nasal cartilage as the best site for the accelerometric measure of nasality. High correlation (.92) has been reported between the accelerometric measure of vibration at the nasal area and the perceptual hypernasality scores (Horii, 1983).

In this study, the term resonant voice is defined as a clear voice production that is easy to produce and is vibrant in the face and oral cavity following Titze's ( 2001) description. One may argue that such definition is purely subjective in nature with both the 'clear voice' and the 'vibrant' sensation as non-objective and non-quantitative perceptions. Nevertheless, these two perceptions are currently the basic components used in the literature to describe and teach resonant voice (see Behrman and Haskell, 2008; Verdolini, 2000). A quantitative definition would have been more appropriate, however, one can only rely on a perceptual definition until there is more evidence on how resonant voice can be measured quantitatively.

The present study was set to answer two research questions: 1) Whether the magnitude of facial bone vibration correlated with the magnitude of auditory-perceptual resonant voice; 2) Whether the use of resonant voice, when compared to a habitual ordinary voice, would increase the magnitude of facial bone vibration.

For the first research question, it was hypothesized that the extent of facial bone vibration correlated positively with the degree of perceptual resonance. For the second research question, it was hypothesized that the resonant voice, learned through training, could increase the extent of facial bone vibration. 


\section{Method}

\section{Participants}

A total of 36 participants (18 females and 18 males with a mean age of 22.67 years $(S D=2.78$, range $=20-33$ years $))$ were recruited. All participants were native Cantonese speakers with no prior experience in singing or voice training. They all reported to be medically healthy, have normal hearing, no history of speech or voice disorders and no prior experience with the use of an accelerometer. The participants were all recruited using the snowball sampling method from the social circles of two authors (GL and GP) on a voluntarily basis.

\section{Procedures}

Resonant voice training and the recordings of bone vibration during resonant voice production were all completed within one session:

Resonant voice training. The training instructions and demonstrations were based on the therapy programs developed by Verdolini (2000) and Yiu and Ho (2002). As mentioned earlier, the definition of resonant voice used in the present study highlighted 1) easy production of clear voice, and 2) vibrant sensation in the face and oral cavity. Participants were taught to concentrate on the auditory-sensory feedback of clear voice production and vibrant sensation which were related to the pitch and amplitude levels. The participants were instructed that they should not feel any tension in the neck when producing resonant voices. Strained voice was also taught and produced as a form of negative practice. The participants were instructed to "strain their neck muscles" in order to produce strained voices. An examiner (either GL or GP) also used the presence or absence of observable straining of neck muscles to determine whether acceptable resonant voice or strained voice was produced. All participants produced the two types of voices as determined by themselves and the examiner without any difficulty. All the subjects were instructed to produce their voice for recording at their comfortable pitch and loudness levels. Details of the training are provided in the Appendix. All recordings were carried out in a 
sound-treated room with background noise kept below 35 dBA. Participants were seated comfortably at an upright position throughout the study.

Vibration and voice recordings. A total of three Brüel \& Kjær's piezoelectric DeltaTron ${ }^{\circledR}$ accelerometers (Type 4507-B-002, Denmark), each with a dimension of $1 \mathrm{x} 1 \mathrm{x} 1 \mathrm{~cm}$, were used to measure the bone vibration (Figure 1). Each accelerometer was $4.8 \mathrm{~g}$ with a frequency range of $0.4 \mathrm{~Hz}$ to $6 \mathrm{kHz}$, resonance frequency of 17.7 $\mathrm{kHz}$, amplitude slope of $-2.4 \% /$ decade, high pass cut off frequency at $0.097 \mathrm{~Hz}$, and low pass cut off frequency at $1430 \mathrm{kHz}$. The vibration captured was amplified using the Nexus ${ }^{\text {TM }}$ conditioning amplifier (Type 2693, Brüel \& Kjær, Denmark) and analyzed using the statistical analysis of the PowerLab Chart software (v 5.4.2, Power Lab AD Instruments, Melbourne).

One of the accelerometers was placed on the forehead at $1 \mathrm{~cm}$ above the midline of the eyebrows. The second one was placed at the right nasal bridge bone just above the septal cartilage. The third accelerometer was placed at the laryngeal prominence of the thyroid cartilage (peri-laryngeal area) to record vibration around the source at the vocal folds (see Figure 2).

\section{Put Figures $1 \& 2$ about here}

Three sets of productions were recorded. One set was recorded before the voice training (pre-training) using a habitual voice and two sets were recorded after the voice training, with one involving the production of resonant voice and the other involving the production of strained voice. Each recording task required the participant to produce the following sounds: 1) two nasal sounds (/ma/ and $/ \mathrm{mi} /) ; 2$ ) two non-nasal sounds (/pa/ and /pi/) (each for four seconds long at the participants' most comfortable pitch and loudness levels); 3) one sentence loaded with nasal consonants (nasalized sentence) /ma ma mo mau/ (meaning "mother touches the cat") and 4) one sentence loaded with non-nasalized sounds /pa pa ta po/ (meaning "father hits the ball").

For each set of recordings, four trials were produced for each sound and sentence. Therefore, each participant produced a total of 72 voice samples ([2 nasal sounds +2 
non-nasal sounds +1 nasal sentence +1 non-nasal sentence] $\times 4$ repetitions $\mathrm{x} 3$ types of voice production (i.e. pre-training habitual non-resonant voice, post-training resonant voice and strained voice)). The order of the trials produced by each participant was counterbalanced across the three types of voice production recording to take into consideration any potential order effects related to the stimuli.

Audio recordings were also taken simultaneously in addition to the accelerometer recordings. All the voice samples were recorded and digitized (at $44.1 \mathrm{kHz}$ ) for perceptual analysis using Dr Speech version 4 (Tiger DRS Inc.) with an AKG Acoustics C420 head-mounted condenser microphone at a 10-cm mouth-to-microphone distance connected to a Creative Extigy USB digital audio signal processor.

\section{Data analysis}

Perceptual rating. Two speech pathologists, each with at least 2 years of experience in assessing and managing voice patients on a daily basis, were recruited as judges for the perceptual rating of the voice samples. They first had to listen once to a set of 36 training voice samples recorded from five male and six female normal speakers. These training voice samples were selected by three of the authors (EY, GL and GP) by inferring the perception of easy phonation and vibrancy (Titze, 2001) by the speakers of these voice samples. These training samples covered a range of minimum to prominent resonance characteristics, as determined and agreed by the three authors EY, GL and GP. The two judges were asked to familiarize themselves with the range of resonance prominence in these training samples but were not required to rate the resonance of these training samples.

In the perceptual rating tasks, two recordings of $/ \mathrm{ma} /$ were randomly selected from the four samples produced by each speaker using the resonant voice. A total of 72 samples (two from each speaker) were prepared. Each judge undertook the procedure individually in a sound-treated room with the 72 samples presented in a random order. The judges could listen to each sample as many times as necessary and were asked to rate the magnitude of resonance of each voice sample on an 11-point 
equal-appearing interval (EAI) scale (Yiu \& Ng, 2004) with “0” representing no resonance and "10" representing prominent resonance. An 11-point EAI scale was used as it is contended to be more sensitive than the more commonly used 7-point EAI scale ( Yiu \& Ng, 2004). Eight (10\%) of the 72 voice samples were randomly selected and were rated again on a second occasion on the same day to obtain the intra-rater agreement. Spearman rho correlations were calculated between the vibration measurements and the auditory-perceptual resonance ratings of the two judges.

Vibration measurement. Data on the bone vibration measured from the nasal bridge and peri-laryngeal sites were analyzed and compared. Data from the forehead site was not analyzed as it was used as a control site for any possible placebo effect. For each vibration, three seconds of the signals that included the consonant onset of each sound or sentence stimulus were extracted and analyzed for the root mean square millivolt (RMS mV). All the nasal sounds $/ \mathrm{ma} /, / \mathrm{mi} /$ and the nasalized sentence were combined to provide a nasal stimulus data set, while the non-nasalized sounds /pa/, /pi/ and the non-nasalized sentence were pooled together to form the non-nasal stimulus data set. It is understood that the bone vibration measured may covary with the overall intensity of the voice production, which hypothetically correlates with the vibration at the laryngeal level. Therefore, the vibration measured at the nasal bridge was normalized against those at the peri-laryngeal area. Thus, the normalized nasal bridge vibration was taken as the ratio between the measures at the nasal bridge and the peri-laryngeal site.

Statistical analysis of vibration data. Two sets of analyses were carried out. First, the vibration data (RMS $\mathrm{mV}$ ) for each stimulus data set was used as the dependent variable in a three-way ANOVA (Stimulus Type [nasal and non-nasal stimuli] $\mathrm{x}$ Voice Type [non-resonant voice, resonant voice, and strained voice] x Site [nasal bridge and peri-laryngeal area]). Second, a two-way ANOVA was conducted on the normalized data (vibration at the nasal bridge normalized against that at the peri-laryngeal area) with the Stimulus Type (nasal and non-nasal stimuli) x Voice Type (non-resonant voice, resonant voice, and strained voice). Multivariate analysis 
of variance using Pillai's criterion was used as it is more robust than the other multivariate statistics in accommodating possible violations of homogeneity of variance-covariance matrices (Max \& Onghena, 1999; Tabachnick \& Fidell, 2001).

\section{Results}

\section{Auditory-perceptual rating of resonance}

The inter-rater agreement value within one point on the perceptual ratings between the two judges were $46.3 \%$, whereas the intra-rater agreement values within one point were $83.7 \%$ and $95.4 \%$. Figure 3 shows the plots of vibration measures $(\mathrm{RMS} \mathrm{mV})$ at the nasal bridge and the peri-laryngeal areas against the perceptual resonance ratings. The Spearman rho correlation of the nasal bridge vibration and mean auditory-perceptual resonance ratings of the two judges was $0.712(\mathrm{p}<0.0001)$, while those of the peri-laryngeal area was $0.622(p<0.0001)$. The correlation of the normalized nasal bridge vibration (against those at the peri-laryngeal area) and the mean auditory-perceptual resonance ratings was nevertheless low (Spearman rho $=-0.135, \mathrm{p}=0.265)$.

\section{Put Figure 3 about here}

\section{Vibration Measurements}

Figure 4 shows the boxplots of the vibration measurements (RMS mV) at each site while Figure 5 shows the normalized vibration values at the nasal bridge.

Main effect of Stimulus, Voice Type, and Site. From Figure 4, it can be seen that the median vibrations with the nasal stimuli in general are all higher than those with the non-nasal stimuli (see Figure 4), regardless of the site of measurement. This was supported by a significant main Stimulus effect $(F=47.97, d f=1, p<.0001)$. The results also showed a significant main effect of Voice Type $(F=8.29, d f=2.00$, $p=.001)$. Planned contrasts showed that all three voice types were significantly different from one another (non-resonant versus resonant: $F=17.08, d f=1, p<.0001$; non-resonant versus strained: $F=4.78, d f=1, p=.036$; resonant versus strained: $F=$ 4.94, $d f=1, p=.033)$. The main effect of Site, however, did not reach a significant 
level $(F=1.90, d f=1, p=.177)$, suggesting there was no significant difference in the vibration between the two sites (nasal bridge and peri-laryngeal area) in general.

Main effect of Stimulus and Voice Type for normalized data. The normalized nasal bridge data show similar results (Figure 5). The nasal stimuli showed higher normalized values than the non-nasal stimuli (Main Stimulus effect: $F=33.92, d f=1$, $p<0.0001)$ and a significant result was also found for the main Voice Type effect $(F=$ $8.075, d f=2, p=0.001)$. Planned contrasts showed the resonant voice type produced significantly higher normalized values than those of the non-resonant voice $(F=$ $16.599, p<0.0001)$ and the strained voice $(F=10.18, p=0.003)$. There was, however, no significant difference between those produced by resonant voice and strained voice $(F=3.79, p=0.59)$.

\section{Put Figures 4 and 5 about here.}

Interaction effects. A significant interaction effect was found with the Stimulus $\mathrm{x}$ Site $(F=19.28, d f=1, p<.0001)$. It can be seen that the nasal stimuli demonstrated relatively little changes across the nasal bridge and the peri-laryngeal area while the non-nasal stimuli showed significantly greater vibration at the peri-laryngeal area than at the nasal bridge. A significant interaction effect was also demonstrated with the Voice Type $\mathrm{x}$ Site interaction $(F=6.81, d f=2, p=.003)$. The magnitudes of vibration at the peri-laryngeal area were rather similar across the three voice types. However, they were different at the nasal bridge. The resonant voice produced the greatest magnitude of vibration, followed by strained and non-resonant voices at the nasal bridge. Planned contrasts showed that the non-resonant voice showed significantly lower vibration than the strained voice $(F=4.72, d f=1, p=.037)$ and the resonant voice $(F=13.59, d f=1, p=.001)$. No significant difference was found between the resonant and strained voices $(F=3.08, d f=1, p=.09)$. The interaction effect of Voice Type x Stimulus was also significant $(F=3.95, d f=2, p=.029)$. Planned contrasts showed that the interaction effect was only present between the resonant and strained voices $(F=7.77, d f=1, p=.009)$. The normalized vibration 
data at the nasal bridge, however, showed no significant Stimulus x Voice Type interaction effect $(F=1.422, d f=2, p=0.255)$.

\section{Discussion}

\section{Auditory-perceptual resonance and vibration}

The piezoelectric accelerometer, a noninvasive instrument used in this study to measure the extent of bone vibration, provides quantitative evidence that the vibration at the nasal bridge reflects the extent of resonant voice. A significant moderate to high correlation (0.712) existed between the auditory-perceptual rating of resonance and the vibration at the nasal bridge. This finding favors the traditional method in teaching resonant voice, which specifically relies on the physical sensation at the nasal bridge (Chen, et al., 2007; Verdolini, 2000; Yiu, 2008; Yiu \& Ma, 2002). Proprioceptive sensation of resonance is one of the features of resonant voice training (Verdolini, 2000). Individuals are always asked to feel the vibration using their fingers at the nasal bridge. These quantitative data support the traditional method in training resonant voice by feeling the vibration at the nasal bridge.

\section{Use of voice nasal stimulus in resonant voice training}

Natural humming or the nasal sound $/ \mathrm{m} /$ are generally used in resonant voice training. This is based on the assumption that these nasal stimuli could facilitate the acquisition of resonant voice (Boone et al., 2005). The nasal stimuli provide greater vibration during resonant voice production. The significant main effect of the Stimulus also supported the traditional use of nasal stimuli in resonant voice training as appropriate. Theoretically, since the acoustic pressure is greater in the comparatively smaller area of nasal cavity than in the vocal tract, greater vibration of nasal stimuli could therefore be detected more readily than that of the non-nasal stimuli during phonation (Titze, 2001). Undoubtedly, in clinical practice, the issue of generalization is important and it is necessary for the individuals to be able to transfer the resonant voice to other speech productions that are used in daily life. 


\section{Resonant versus strained phonation}

It should be noted that the significant main effect of Voice Type found that resonant phonation produced the greatest vibration while strained phonation also produced significantly greater vibration than non-resonant phonation, but not as much as that produced by the resonant phonation. Indeed, it is possible to produce a greater vibration at the nasal bridge by using a stronger intensity through straining voice. Indeed, our normalized data showed that no significant difference was found between the normalized resonant voice and strained voice data $(F=3.79, p=0.59)$. This suggests that the extra effort at the laryngeal level could produce equally higher vibration at the nasal bridge.

Resonant voice phonation uses easy phonation that can reduce the tension of the extrinsic laryngeal muscles (Harris, Harris, Rubin, \& Howard, 1998). In contrast, strained voice would increase the tension in laryngeal muscles. Hence, it is suggested that the vibratory sensation at the nasal bridge should be emphasized in resonant voice training.

Nevertheless, the effect of proprioceptive feedback on learning to produce resonant voice was not investigated in the present study. Learners were often asked to monitor their voice by both auditory and proprioceptive feedback during resonant voice training. This study found that the perceptual rating of resonance would not be easy. The two experienced judges demonstrated high intra-rater reliability but relatively low inter-rater reliability. Further study on the comparison of these two types of feedback during resonant voice training would be warranted.

It should also be noted that the resonant voice training session used in the present study was only for 30 minutes. This was not a typical representation of traditional resonant voice training, which usually covers more than one session (Chen, et al., 2007; Verdolini, 2000; Yiu, 2008; Yiu \& Ma, 2002). 


\section{Conclusion}

The present study supported the use of a piezoelectric accelerometer as a noninvasive instrument to acquire a quantitative measure of the magnitude of vibration of the nasal bone and peri-laryngeal area associated with phonation produced by individuals with normal voice. Significantly greater vibration with nasal stimuli than with non-nasal stimuli was detected in the study. Furthermore, the results of the study demonstrated that the agreement of auditory-perceptual judgment of resonance is highly variable across judges. This would therefore limit the widespread use of the auditory-perceptual method. Therefore, it is necessary to find a reliable and easier means to judge resonance. The effectiveness of visual feedback provision in resonant voice training would need to be extended to the dysphonic population to investigate the effectiveness of this quantitative measurement of vibration. 


\section{Reference}

Barrichelo, V. M., \& Behlau, M. (2007). Perceptual identification and acoustic measures of the resonant voice based on "Lessac's Y-Buzz"- a preliminary study with actors. Journal of Voice, 21(1), 46-53.

Behrman, A., \& Haskell, J. (2008). Resonant Voice. In A. Behrman \& J. Haskell (Eds.), Exercises for voice therapy (pp. 47-53). San Diego: Plural Publishing.

Boone, D. R., McFarlane, S. C., \& Von Berg, S. L. (2005). The voice and voice therapy $\left(7^{\text {th }}\right.$ ed.). Pearson/Allyn \& Bacon.

Chen, S. H., Hsiao, T. Y., Hsiao, L. C., Chung, Y. M., \& Chiang, S. C. (2007).

Outcome of resonant voice therapy for female teachers with voice disorders:

Perceptual, physiological, acoustic, aerodynamic, and functional

measurements. Journal of Voice, 21(4), 415-425.

Chen, S. H., Huang, J., \& Chang, W. (2003). The efficacy of resonance method to hyperfunctional dysphonia from physiological, acoustic and aerodynamic aspects: The preliminary study. Asia Pacific Journal of Speech, Language and Hearing, 8, 200-203.

Colton, R. H., \& Casper, J. (1996). Understanding Voice Problems: A Physiological Perspective for Diagnosis and Treatment. New York: Williams \& Wilkins.

Cooper, M., \& Cooper, M. H. (1977). Approaches to ocal ehabilitation. Springfield, IL: C. C. Thomas.

Harris, T., Harris, S., Rubin, J. S., \& Howard, D. M. (1998). The voice clinic handbook. London: Whurr Publishers Ltd.

Horii, Y. (1983). An accelerometric measure as a physical correlate of perceived hypernasality in speech. Journal of Speech and Hearing Research, 26(3), 476-480.

Jacobson, B. H., \& White, J. P. (1994). Multidisciplinary approach to treatment. In M. S. Benninger, B. H. Jacobson \& A. F. Johnson (Eds.), Vocal arts medicine: The care \& prevention of professional voice disorders (pp. 318-343). New 
York: Thieme Medical Publishers, Inc.

Kreiman, J., Gerratt, B. R., Kempster, G. B., Erman, A., \& Berke, G. S. (1993).

Perceptual evaluation of voice quality: review, tutorial, and a framework for future research. Journal of Speech and Hearing Research, 36, 21-40.

Lippmann, R. P. (1981). Detecting nasalization using a low-cost miniature accelerometer. Journal of Speech and Hearing Research, 24(3), 314-317.

Max, L., \& Onghena, P. (1999). Some issues in the statistical analysis of completely randomized and repeated measures designs for speech, language, and hearing research. Journal of Speech, Language and Hearing Research, 42, 261-270.

Peterson, K. L., Verdolini-Marston, K., Barkmeier, J. M., \& Hoffman, H. T. (1994). Comparison of aerodynamic and electroglottographic parameters in evaluating clinically relevant voicing patterns. Annals of Otology, Rhinology and Laryngology, 103, 335-346.

Roy, N., Weinrich, B., Gray, S. D., Tanner, K., Stemple, J. C., \& Sapienza, C. M. (2003). Three treatments for teachers with voice disorders: a randomized clinical trial. Journal of Speech, Language, and Hearing Research, 46(3), $670-688$.

Shrivastav, R., Sapienza, C. M., \& Nandur, V. (2005). Application of psychometric theory to the measurement of voice quality using rating scales. Journal of Speech, Language, and Hearing Research, 48(2), 323-335.

Stevens, K. N., Kalikow, D. N., and Willemain, T. R. (1975). A miniature accelerometer for detecting glottal waveforms and nasalization. Journal of Speech and Hearing Research, 18(3), 594-599.

Sundberg, J. (1983). Chest wall vibrations in singers. Journal of Speech and Hearing Research, 26, 329-340.

Svec, J. G., Titze, I. R., \& Popolo, P. S. (2005). Estimation of sound pressure levels of voiced speech from skin vibration of the neck. Journal of Acoustical Society of America, 117(3), 1386-1394. 
Tabachnick, B. G., \& Fidell, L. S. (2001). Multivariate analysis of variance and covariance. In B. G. Tabachnick \& L. S. Fidell (Eds.), Using Multivariate Statistics (pp. 375-440). Boston: Allyn and Bacon.

Titze, I. R. (2001). Acoustic Interpretation of Resonant Voice. Journal of Voice, 15(4), $519-528$.

Verdolini, K. (1998). Resonant voice therapy. In K. Verdolini (Ed.), Voice and speech's guide to vocology (pp. 34-35). Iowa City, IA: National Center for Voice and Speech.

Verdolini, K. (2000). Case study: Resonant voice therapy. In J. Stemple (Ed.), Voice therapy: Clinical studies (2nd ed., pp. 46-62). San Diego, CA: Singular Publishing Group.

Verdolini, K., Druker, D. G., Palmer, P. M., \& Samawi, H. (1998). Laryngeal adduction in resonant voice. Journal of Voice, 12(3), 315-327.

Verdolini, K., Hess, M. M., Titze, I. R., Bierhals, W., \& Gross, M. (1999). Investigation of vocal fold impact stress in human subjects. Journal of Voice, 13(2), 184-202.

Verdolini-Marston, K., Burke, M. K., Lessac, A., Glaze, L., \& Caldwell, E. (1995). Preliminary study of two methods of treatment for laryngeal nodules. Journal of Voice, 9(1), 74-85.

Yiu, E. M.-L. (2008). Resonant voice: Hong Kong humming. In A. Behrman \& J. Haskell (Eds.), Exercises for voice therapy (pp. 62-68). San Diego: Plural Publishing.

Yiu, E. M.-L., \& Ho, E. Y.-Y. (2002). Short-term effect of humming on vocal quality. Asia Pacific Journal of Speech, Language and Hearing, 7, 123-137.

Yiu, E. M.-L., \& Ma, E. P. (2002). Voice activity limitation and participation restriction in teaching profession: the need for preventative care. Journal of Medical Speech-Language Pathology, 10(1), 51-60.

Yiu, E. M.-L., \& Ng, C. Y. (2004). Equal appearing interval and visual analogue scaling of perceptual roughness and breathiness. Clinical Linguistics \& Phonetics, 18(3), 211-229. 
Acknowledgement: This paper was prepared while the first author took his sabbatical at the Faculty of Health Sciences, The University of Sydney. Research support was given to the author by the Dean, Professor Gwynnyth Llewellyn and her staff to enable the completion of this manuscript. 
Appendix

Instructions of resonant voice training (Yiu \& Ho, 2002)

1) The participant is reminded to sit at a relaxed manner throughout the training.

2) Say "uh-um" or $/ \mathrm{m} /$ softly as in acknowledging someone asking a question. The hum has to be produced in a relaxed manner. Demonstration can be provided by the trainer.

3) Glide $/ \mathrm{m} /$ up and down a musical scale to find a pitch that resultes in maximum resonance/vibration without straining of laryngeal muscles. The located pitch will then be used as the note for further humming. Demonstration will be provided by the trainer.

4) Put a finger on the nasal bridge to feel the vibration. The kinesthetic feedback of possible tingling sensation around the lip area is also explained.

5) Listen to the voice quality of the hum with comments on the performance given by the trainer. Feedback related to the participant's gentle onset, resonance, and comfortable amplitude and pitch level should be provided.

6) Hum at a comfortable pitch in a relaxed manner and add a sustained vowel to the end of the $/ \mathrm{m} /$. Smooth transition from the sound $/ \mathrm{m} /$ to $/ \mathrm{a} /$ is emphasized. Demonstration can be provided by the trainer.

7) Listen to the voice quality of /ma/ with comments on the performance given by the trainer. 


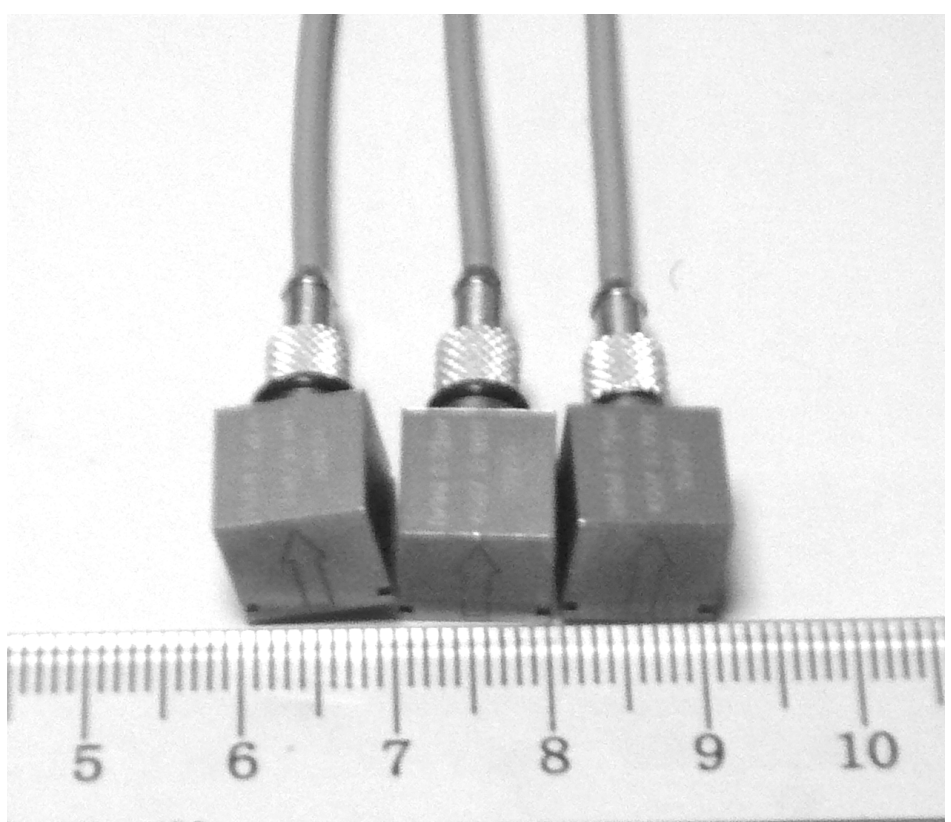

Figure 1. Piezoelectric accelerometers (Miniature DeltaTron ${ }^{\circledR}$ accelerometer) 
Nasal

bridge
Forehead

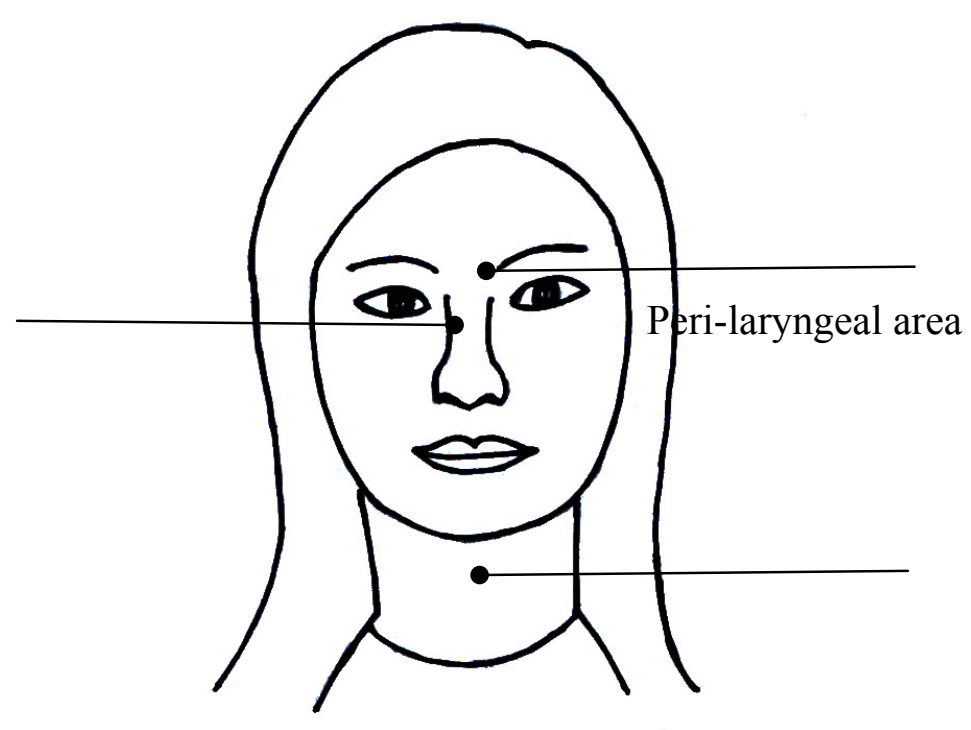

Figure 2. Placement of accelerometers at the forehead, the nasal bridge, and the peri-laryngeal area. 

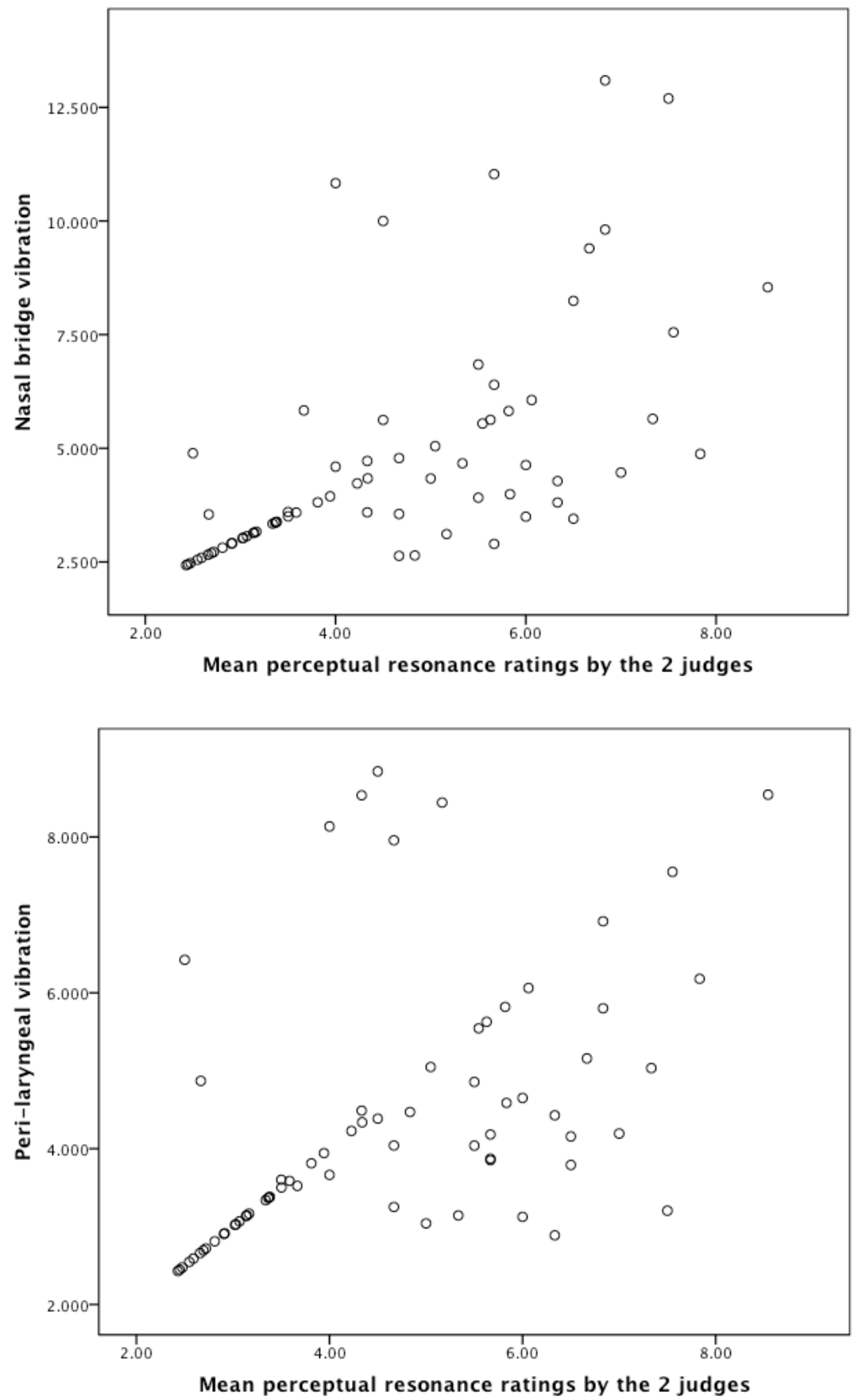

Figure 3. Vibration (RMS mV) plot against perceptual resonance ratings 




- Midline of the box is the median. The bottom and top of the box show the 25 th and 75 th percentile (the lower and upper quartiles).

- The top and bottom lines represent the highest and lowest values that are not outliers (i.e. within 1.5 times the interquartile range).

- The outliers (between 1.5 and 3 times the interquartile range) are represented by circles, and extreme values (more than 3 times the interquartile range) are represented by asterisks.

Figure 4. Vibration (RMS mV) during phonation. 


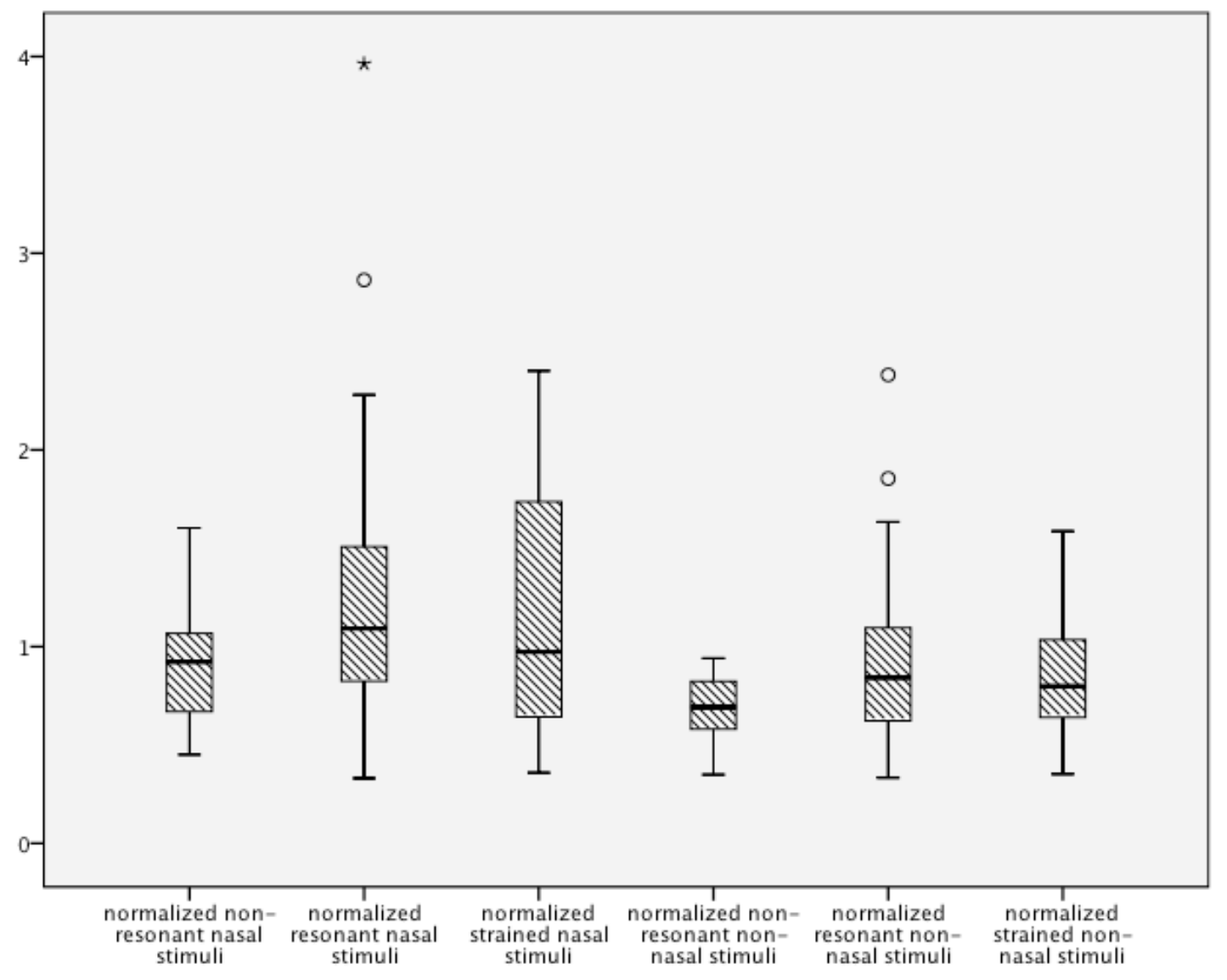

- Midline of the box is the median. The bottom and top of the box show the 25 th and 75 th percentile (the lower and upper quartiles).

- The top and bottom lines represent the highest and lowest values that are not outliers (i.e. within 1.5 times the interquartile range).

- The outliers (between 1.5 and 3 times the interquartile range) are represented by circles, and extreme values (more than 3 times the interquartile range) are represented by asterisks.

Figure 5. Normalized vibration (RMS $\mathrm{mV}$ ) at nasal bridge against peri-laryngeal area during phonation. 